\title{
Influence of municipal wastewater on rice seed germination, seedling performance, nutrient uptake, and chlorophyll content
}

\begin{abstract}
The sand medium experiment was conducted in the laboratory with the aim of evaluating the suitability of municipal wastewater (treated and untreated) at different concentrations $(0,2.5$, $5,10,25,50$, and $100 \%$ ) for irrigation purposes on seed germination, seedling performance, nutrient uptake, and chlorophyll content of rice (MR220). The utilization of municipal wastewater for irrigation purposes has developed an important way to exploit the nutrients it contains. The study indicated a significant $(\mathrm{P}<0.05)$ difference between untreated and treated municipal wastewater for seedling length (SL), root volume (RV), root surface area (RSA), seedling vigor index (SVI), and root: shoot ratio (R : S) while no significant different was observed between untreated and treated municipal wastewater for germination percentage (GP) and percentage phytotoxicity (PPT). The municipal wastewaters had stimulatory effect on the rice seeds at lower wastewater concentrations $(<25 \%)$ while inhibitory effect was observed at higher wastewater concentrations (> 50\%). N, P, K, Ca, Mg, $\mathrm{Zn}, \mathrm{Fe}, \mathrm{Cu}$, and $\mathrm{Mn}$ were high in the untreated municipal wastewater compare to treated municipal wastewater. Seedlings irrigated with untreated municipal wastewater have high seed germination, seedling performance with high nutrient uptake compare to treated municipal wastewater. The municipal wastewaters showed inhibitory effect on chlorophyll content. The inhibition effect was observed at $>50 \%$ concentration of both untreated and treated municipal wastewater while promoting effects were observed at lower $(<25 \%)$ concentration. The rice MR220 variety showed better growth resistance, tolerance, and adaptability to wastewater. This can be due to its excellent genetic makeup and capacity to sustain the toxicity. Conclusively, municipal wastewater could be recommended as a good source of water and nutrient for seed germination and seedling performance.
\end{abstract}

Keyword: Chlorophyll content; Municipal wastewater; Nutrient uptake; Oryza sativa; Seed germination; Seedling performance 